\title{
Cusp Catastrophe Model for Description of Bistability in Ferroelectric Liquid Crystals
}

\author{
Nail Migranov, ${ }^{1}$ Aleksey Kudreyko, ${ }^{2}$ and Denis Kondratyev ${ }^{3}$ \\ ${ }^{1}$ Department of General and Theoretical Physics, Bashkir State Pedagogical University, Okt. Revolutsii Street 3A, \\ Ufa 450000, Russia \\ ${ }^{2}$ Department of Physics, Ufa State Petroleum Technological University, Kosmonavtov Street 1, Ufa 450062, Russia \\ ${ }^{3}$ Academy of Sciences of Bashkortostan, Kirova Street 15, Ufa 450008, Russia
}

Correspondence should be addressed to Aleksey Kudreyko; akudreyko@rusoil.net

Received 4 June 2014; Accepted 21 August 2014; Published 1 September 2014

Academic Editor: Anand Pathak

Copyright (C) 2014 Nail Migranov et al. This is an open access article distributed under the Creative Commons Attribution License, which permits unrestricted use, distribution, and reproduction in any medium, provided the original work is properly cited.

We propose a new theoretical approach for description of the equilibrium and bistable states of ferroelectric liquid crystal. A sample of ferroelectric liquid crystal is considered in the "bookshelf" geometry under the influence of electric field. Continuum and perturbation theories are used in order to establish the relation between the scale coefficients in the expansion for the azimuthal angle. The equilibrium states of ferroelectric $\mathrm{SmC}^{*}$ are determined in the framework of the catastrophe theory.

\section{Introduction}

Ferroelectric liquid crystals (FLCs) are widely used in production of fast displays in electronic equipment due to fast response time $(\sim 100 \mu s)$ to the electric field. Inherent permanent polarization that interacts with the electric field makes ordering of molecules completely different from usual liquid crystals, and in this connection numerous technical applications arouse great interest in investigations of FLC structures. In 1980 Clark and Lagerwall [1] introduced bistable FLC. These crystals are considered as a potential operating component for production of e-paper due to its fast response time, wide viewing angle, and so forth [2].

However, the progress in the development of optical processing systems is being impeded by the lack of enough knowledge on instabilities of FLCs, which is related with the free energy. One of the main advantages in the application of the methods of bifurcation theory to nonlinear stability problems is that in general it is possible to reduce the dimension of the original system to a low dimensional bifurcation system, mainly in the neighborhood of the critical points.

As known, bifurcation theory and catastrophe theory are two of the best known areas in the field of dynamical systems. Due to its topological character, catastrophe theory has several advantages that allow getting qualitative results. Catastrophe models have been successfully applied in various fields of research [3]. Gibelli and Turzi [4] applied catastrophe theory to derive a criterion for identification of tricritical points in a multiorder parameter system represented by SmC. In $[5,6]$ authors proposed that the Landau expansion of free energy can be represented as a catastrophe functional that possesses inherent properties for description of instabilities. The lack of this proposition is that the corresponding relation between the behavior parameters is not represented.

In our research we applied asymptotic methods in order to elaborate the relation of the governing parameters in the series of azimuthal angle. Due to the complexity of the torque balance equation, it is difficult to find the values of the governing parameters and calculate the minima of free energy. The applied catastrophe theory to the study of the free energy potential of FLC provided a detailed mathematical description of how the geometry of the energy functional varies with the control parameters. The local geometry around the critical points of the free energy functional is represented by a certain catastrophe function, which is determined by the elastic, surface, spontaneous polarization and dielectric free energy densities. The obtained plots confirm 


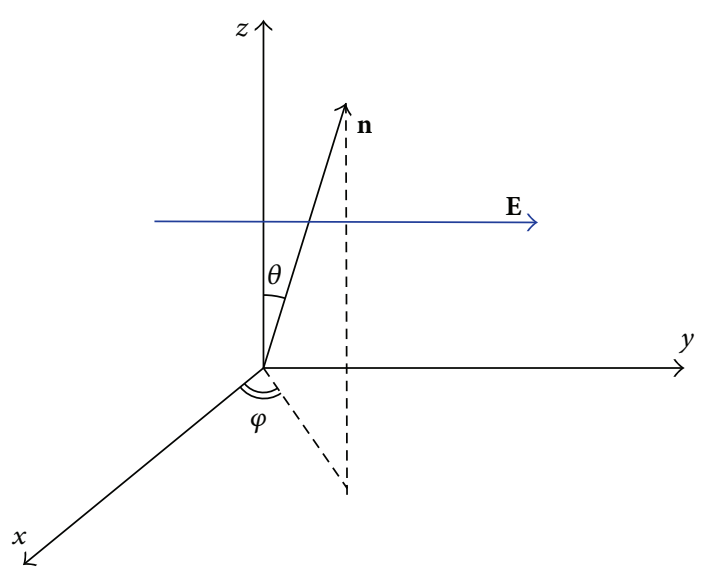

FIGURE 1: Schematic representation of the director $\mathbf{n}$ in the electric field $\mathbf{E}$.

the validity of the catastrophe theory for description of bistability of FLC. The advantage of this qualitative approach is that the catastrophe functional makes it possible to find energy minima and bifurcation points in multiparameter dynamic systems.

This paper is organized as follows: in Section 2 we introduce the relation that models the behavior of the azimuthal angle and free energy of ferroelectric liquid crystal. Then with the aid of the torque balance equation and the approximation theory we establish the relation between the behavior parameters in the proposed relation. In Section 3 we introduce the normalized free energy potential in the form of the cusp catastrophe. The results are discussed in Section 4.

\section{Free Energy of Ferroelectric Liquid Crystal}

Assume that in the absence of the electric field molecules of FLC align in parallel to a substrate (planar alignment). When electric field is turned on, consider a director $\mathbf{n}$ of FLC, which is placed in this field

$$
\mathbf{n}=\sin \theta \cos \varphi \mathbf{e}_{x}+\sin \theta \sin \varphi \mathbf{e}_{y}+\cos \theta \mathbf{e}_{z}
$$

where $\theta$ is the polar angle between $\mathbf{n}$ and the $z$-axis and $\varphi$ is the azimuthal angle in the Cartesian coordinate system. As illustrated in Figure 1, the electric field $\mathbf{E}$ in the FLC is directed along the $y$-axis, which is normal to the bounding plates separated by a distance $d$, and the sample of FLC is uniform along the $x$ - and $z$-axes.

The total free energy density $G$ of FLC, which is confined between two plates, consists of elastic energy density $F_{\mathrm{el}}$, surface energy density $F_{s}$, spontaneous polarization density $F_{p}$, and the dielectric interaction energy density $F_{\text {diel }}$ induced by the applied field. Then the total free energy $G$ of the FLC is given by

$$
G(\varphi)=\int_{V}\left(F_{\mathrm{el}}+F_{p}+F_{\mathrm{diel}}\right) d V+\int_{S} F_{s} d S
$$

where $S, V$ are area and volume of the sample. The substitution of $\mathbf{n}$ into the elastic part of the free energy density gives

$$
\begin{aligned}
F_{\mathrm{el}}= & F_{0}+\frac{1}{2} A\left(1+\nu \sin ^{2} \varphi\right)\left(\varphi_{, y}\right)^{2} \\
& +A(1+\nu) \varphi, y Q_{0} \sin \varphi,
\end{aligned}
$$

where

$$
\begin{gathered}
F_{0}=\frac{1}{2}\left(K_{2} q_{T}^{2}+K_{3} q_{B}^{2}\right), \\
A=K_{1} \sin ^{2} \theta, \quad B=\left(K_{2} \cos ^{2} \theta+K_{3} \sin ^{2} \theta\right) \sin ^{2} \theta, \\
\nu=\frac{B-A}{A}, \quad Q_{0}=\frac{K_{2} q_{T} \sin \theta \cos \theta+K_{3} q_{B} \sin ^{2} \theta}{A(1+\nu)} .
\end{gathered}
$$

In these expressions $q_{T}$ and $q_{B}$ are the inherent twist and bend wavenumbers $\left(\simeq 1.3 \cdot 10^{6} \mathrm{~m}^{-1}\right) ; K_{1}, K_{2}, K_{3}$ are splay, twist, and bend elastic constants.

The dielectric interaction energy density $F_{\text {diel }}$ is expressed as

$$
F_{\text {diel }}=-\frac{1}{2} \varepsilon_{\perp} \varepsilon_{0}\left(1+\Delta \varepsilon^{\prime} \sin ^{2} \varphi\right) E^{2},
$$

where $\Delta \varepsilon^{\prime}=\left(\varepsilon_{\|}^{\prime}-\varepsilon_{\perp}\right) / \varepsilon_{\perp}, \varepsilon_{\|}^{\prime}=\varepsilon_{\perp} \cos ^{2} \theta+\varepsilon_{\|} \sin ^{2} \theta$, and $\varepsilon_{0}$ is the dielectric constant. Next, the polarization density $F_{p}$ is given by

$$
F_{p}=P_{s} E \cos \varphi,
$$

where $P_{s}$ is the magnitude of the polarization vector; the physical interpretation of $\mathbf{P}_{\mathbf{s}}$ can be found, for example, in [7]. The surface interaction associated with the anchoring is defined as

$$
F_{s}=-g_{1} \cos ^{2}\left(\varphi-\varphi_{s}\right)-g_{2} \cos \left(\varphi-\varphi_{s}\right),
$$

where $g_{1}, g_{2}$ denote azimuthal and polar anchoring strengths between the molecules of liquid crystal and bounding plates and $\varphi_{s}$ is the pretilt angle of the molecules $[7,8]$.

Suppose that $\varphi \simeq 0$ (e.g., $0<\varphi \leq \pi / 36 \mathrm{rad}$ ); then the following approximations are valid:

$$
\begin{aligned}
& \sin \varphi \approx \varphi-\frac{1}{6} \varphi^{3}+\frac{1}{120} \varphi^{5}, \\
& \cos \varphi \approx 1-\frac{1}{2} \varphi^{2}+\frac{1}{24} \varphi^{4} .
\end{aligned}
$$

It is known that in FLCs the spontaneous polarization vector $\mathbf{P}_{\mathrm{s}}$ exhibits inherent rotation within the $x y$-plane. Assume that the mean of the azimuthal angle $\varphi$ is defined relative to the $y$-axis as the following series:

$$
\varphi=\sum_{i=0}^{n} a_{i} \tilde{y}^{i}
$$

where $\tilde{y}$ is the normalized length $\tilde{y}=y / d$ and $a_{i}$ is a dimensionless parameter. Consider this expansion for $n=2$; then

$$
\varphi=a_{0}+a_{1} \tilde{y}+a_{2} \tilde{y}^{2} .
$$


In the following, we will assume that $\tilde{y}=y$; then $y \in[0 ; 1]$. The substitution of (10) and approximations of trigonometric functions into the stationary torque balance equation [7] under the one-constant approximation $\left(K_{1}=K_{2}=K_{3}=K\right)$

$$
K \sin ^{2} \theta \frac{1}{d^{2}} \frac{\partial^{2} \varphi}{\partial y^{2}}+\frac{\Delta \varepsilon^{\prime}}{2} \varepsilon_{\perp} E^{2} \sin 2 \varphi+P_{s} E \sin \varphi=0
$$

yields the following equation for the terms up to $y^{2}$ :

$$
\begin{aligned}
& \left(2 \Delta \varepsilon^{\prime} \varepsilon_{\perp} E^{2} a_{0} a_{1}^{2}-\frac{1}{2} P_{s} E a_{0} a_{1}^{2}+\frac{1}{24} P_{s} E a_{0}^{4} a_{2}\right. \\
& +\frac{2}{3} \Delta \varepsilon^{\prime} E^{2} a_{0}^{4} a_{2}+2 \Delta \varepsilon^{\prime} \varepsilon_{\perp} E^{2} a_{0}^{2} a_{2}+P_{s} E a_{2} \\
& -\Delta \varepsilon^{\prime} \varepsilon_{\perp} E^{2} a_{2}+\frac{4}{3} \Delta \varepsilon^{\prime} \varepsilon_{\perp} E^{2} a_{0}^{3} a_{1}^{2} \\
& \left.+\frac{1}{12} P_{s} E a_{0}^{3} a_{1}^{2}-\frac{1}{2} P_{s} E a_{0}^{2} a_{2} E\right) y^{2} \\
& +\left(-2 \Delta \varepsilon^{\prime} \varepsilon_{\perp} E^{2} a_{0}^{2} a_{1}+\frac{2}{3} \Delta \varepsilon^{\prime} \varepsilon_{\perp} E^{2} a_{0}^{4} a_{1}+P_{s} E a_{1}\right. \\
& \left.\quad-\frac{1}{2} P_{s} E a_{0}^{2} a_{1}+\Delta \varepsilon^{\prime} \varepsilon_{\perp} E^{2} a_{1}+\frac{1}{24} P_{s} E a_{0}^{4} a_{1}\right) y \\
& +\frac{1}{120} P_{s} E a_{0}^{5}+P_{s} E a_{0}+\Delta \varepsilon^{\prime} \varepsilon_{\perp} E^{2} a_{0}-\frac{2}{3} \Delta \varepsilon^{\prime} \varepsilon_{\perp} E^{2} a_{0}^{3} \\
& +\frac{2 K \sin ^{2} \theta}{d^{2}} a_{2}+\frac{2}{15} \Delta \varepsilon^{\prime} \varepsilon_{\perp} E^{2} a_{0}^{5}-\frac{1}{6} P_{s} E a_{0}^{3}=0
\end{aligned}
$$

where $\theta$ is the constant along the $y$-axis and $v=0$.

The complexity of (12) makes it unsolvable analytically with respect to coefficients $a_{i}$. In order to gain some insight into the free energy potential (2), we apply the perturbation methods [9]. Since (12) is an identity for each parameter $y$, then each factor of $y^{i}(i=0,1,2)$ vanishes independently. Consider the factor of $y$ in the identity (12); then we obtain

$$
\begin{aligned}
a_{1}\left(P_{s} E-2 \Delta \varepsilon^{\prime} \varepsilon_{\perp} E^{2} a_{0}^{2}+\frac{2}{3} \Delta \varepsilon^{\prime} \varepsilon_{\perp} E^{2} a_{0}^{4}\right. \\
\left.-\frac{1}{2} P_{s} E a_{0}^{2}+\Delta \varepsilon^{\prime} \varepsilon_{\perp} E^{2}+\frac{1}{24} P_{s} E a_{0}^{4}\right)=0 .
\end{aligned}
$$

This means that either $a_{1}=0$ or

$$
\begin{aligned}
& \left(16 \Delta \varepsilon^{\prime} \varepsilon_{\perp} E^{2}+P_{s} E\right) a_{0}^{4}+\left(12 P_{s} E-48 \Delta \varepsilon^{\prime} \varepsilon_{\perp} E^{2}\right) a_{0}^{2} \\
& +24\left(\Delta \varepsilon^{\prime} \varepsilon_{\perp} E^{2}+P_{s} E\right)=0
\end{aligned}
$$

then

$$
\begin{gathered}
a_{0}= \pm\left(\frac{24 \Delta \varepsilon^{\prime} \varepsilon_{\perp}+6 P_{s}}{P_{s}+16 \Delta \varepsilon^{\prime} \varepsilon_{\perp} E}\right. \\
\left. \pm \frac{2\left(48\left(\Delta \varepsilon^{\prime} \varepsilon_{\perp} E\right)^{2}-30 \Delta \varepsilon^{\prime} \varepsilon_{\perp} P_{s}+3 P_{s}^{2}\right)^{1 / 2}}{P_{s}+16 \Delta \varepsilon^{\prime} \varepsilon_{\perp} E}\right)^{1 / 2} .
\end{gathered}
$$

There exist many FLCs that possess negative and positive dielectric anisotropy [10]. In our example we suppose that $\varepsilon_{\|}=3.89, \varepsilon_{\perp}=2.91, \theta=2 \pi / 15, \varphi_{s}=7 \pi / 60$, and $K=10^{-12} \mathrm{~N}$ [11]. The substitution of the representative magnitudes of the polarization vector and the electric field in (15) $P_{s}=10^{-5} \mathrm{C}$. $\mathrm{m}^{-2}, E=5 \cdot 10^{6} \mathrm{~V} \cdot \mathrm{m}^{-1}$ gives $\left|a_{0}\right| \simeq 1$. This issue violates the condition $\varphi \simeq 0$; therefore $a_{1}=0$. From the free term of $y$ in (12) we find that

$$
\begin{aligned}
a_{2}= & -\frac{a_{0} d^{2} E}{240 K \sin ^{2} \theta} \\
& \times\left(120 \Delta \varepsilon^{\prime} \varepsilon_{\perp} E-80 \Delta \varepsilon^{\prime} \varepsilon_{\perp} E a_{0}^{2}+16 \Delta \varepsilon^{\prime} \varepsilon_{\perp} E a_{0}^{4}\right. \\
& \left.+P_{s} a_{0}^{4}-20 P_{s} a_{0}^{2}+120 P_{s}\right)
\end{aligned}
$$

that is, $a_{2} \simeq-10^{4} a_{0}$ for $d=20 \mu \mathrm{m}$. With the exception of the trivial solution in the factor of $y^{2}$, our further analysis assumes that $\left|a_{0}\right| \ll 1$, so we can neglect it with respect to $a_{2}$.

Combining functionals (3)-(8) in (2), we find that the total free energy $G$ is a function of $y$, where $y \in[0 ; 1]$. Carrying out the integration over $y$, we obtain

$$
G\left(a_{2}\right)=R a_{2}^{4}+S a_{2}^{2}+T a_{2}+U,
$$

where

$$
\begin{aligned}
& R=\frac{1}{54}\left(-\frac{1}{8} P_{s} E+E \Delta \varepsilon^{\prime} \sin ^{2} \varphi_{s}\right)-\frac{1}{24} K Q_{0} \sin ^{2} \varphi_{s}, \\
& S=\frac{1}{10}\left(\frac{1}{2} P_{s} E-\Delta \varepsilon^{\prime} E^{2} \sin ^{2} \varphi_{s}\right)+\frac{1}{2} K Q_{0} \sin ^{2} \varphi_{s}+\frac{2}{3} K \sin ^{2} \varphi_{s}, \\
& T=-\frac{1}{3}\left(g_{2} \sin \varphi_{s}+g_{1} \sin 2 \varphi_{s}\right), \\
& U=F_{0}-\frac{1}{2} E^{2} \varepsilon_{\perp}-\frac{1}{2} P_{s} E .
\end{aligned}
$$

Note that for the given parameters of FLC, the factor of $a_{2}^{3}$ can be neglected because

$$
\frac{1}{21}\left(2 g_{1} \sin 2 \varphi_{s}+\frac{1}{2} g_{2} \sin \varphi_{s}\right) a_{2}^{3} \ll|T| a_{2},
$$

where $g_{1}, g_{2} \simeq 10^{-5} \mathrm{~J} \cdot \mathrm{m}^{-2}\left(\simeq 10^{-2} \mathrm{erg} \cdot \mathrm{cm}^{-2}\right)[12]$; therefore we will not take it into consideration.

\section{Cusp Catastrophe Model}

To get free term in higher degree of functional (17), it is convenient to divide it by $R$. So, we analyze the normalized free energy and have reduced our problem to the famous cusp catastrophe [6]. It is known that two types of variables are distinguished in the catastrophe theory: control and behavior variables. The behavior of the system is described by the relation between these variables.

Free energy $\widetilde{G}$ written in terms of the cusp catastrophe model is

$$
\widetilde{G}\left(a_{2}\right)=a_{2}^{4}+\frac{S}{R} a_{2}^{2}+\frac{T}{R} a_{2}+\frac{U}{R}
$$




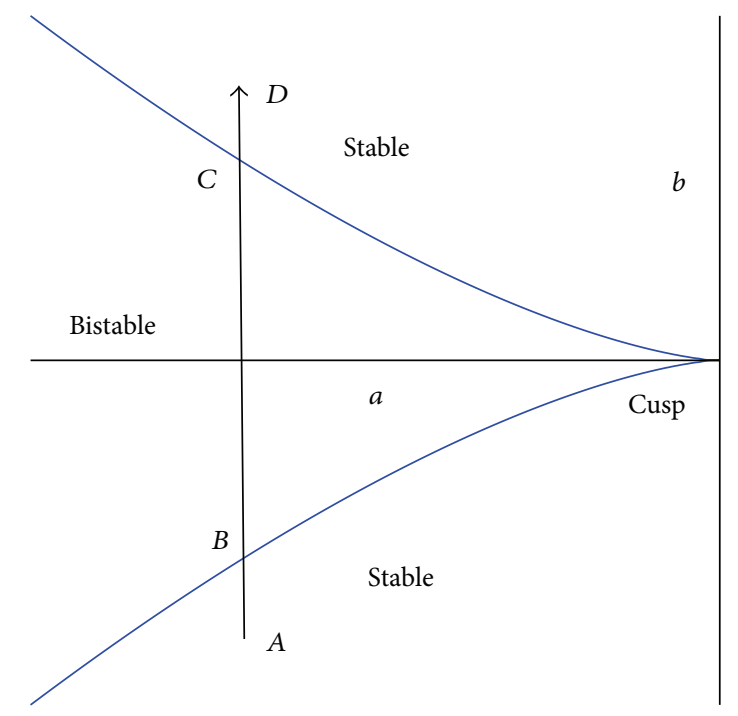

FIGURE 2: Relation between $a$ and $b$, where $a$ changeover takes place from one to two minima in the $\widetilde{G}$ function.

where $a_{2}$ plays a role of the behavior parameter. Therefore, functional (20) can be referred to as bifurcation model exhibiting a cusp catastrophe. Depending on the control parameters $a=S / R$ and $b=T / R$, the $\widetilde{G}$ has one or two minima. The bifurcation set is given by the condition that a critical point is degenerate. Then the projection of $\widetilde{G}$ into the $(a, b)$ space can be determined as

$$
\frac{\partial \widetilde{G}}{\partial a_{2}}=0, \quad \frac{\partial^{2} \widetilde{G}}{\partial a_{2}^{2}}=0
$$

and by eliminating $a_{2}$, we obtain [3] the bifurcation set

$$
\Delta=8 a^{3}+27 b^{2}=0 .
$$

This set is plotted in Figure 2, where two curves merge according to the power-law behavior.

It is clear that the explicit computation of $G\left(a_{2}\right)$ represents a difficulty; therefore we will make a qualitative analysis of (20) and (22). Consider the line $A-B-C-D$ in Figure 2 for a fixed mean of the parameter $a$. In point $A$ only one minimum of free energy exists, and the system is assumed to be in this minimum (solid line in Figure 3). When we increase $b$, the system shifts to the point $B$, where a second minimum starts to appear (dashed line in Figure 3).

When the values of $E$ and $P_{s}$ are such that the state of FLC is between the bifurcation set (line $B C$ ), there exist two isolated minima with a potential barrier between them (dash-dotted line in Figure 3). The barrier disappears in the bifurcation point $C$, and even a small perturbation causes the system to move to the left-hand minimum. Similar behavior is possible when we move from point $D$ to $A$, but when we change the field polarity and follow from $C$ to $B$, FLC enters the right-hand minimum (Figure 3). As can be seen, a hysteresis in FLC occurs exhibiting free-energy wells. In other words, the behavior of FLC depends on the direction of the way that the system changes.

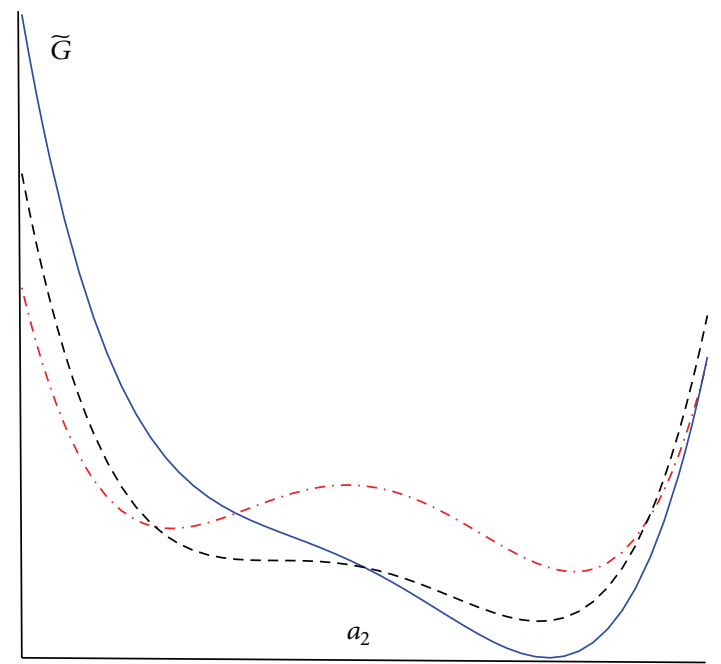

FIGURE 3: Schematic representation of $\widetilde{G}$ versus different means of the control parameters $a, b$.

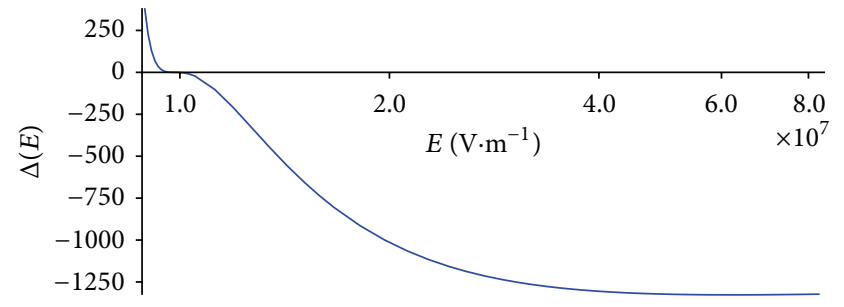

Figure 4: Cross section of $\widetilde{G}(E)$ for $P_{s}=2 \cdot 10^{-5} \mathrm{C} \cdot \mathrm{m}^{-2}$.

The explanation of this effect is known in literature as the Clark-Lagerwall bistability $[1,10]$, which occurs when electric field breaks zenithal anchoring. In the surface stabilized FLC geometry, the free-energy wells (Figure 3) at two azimuthal orientations of the director on the tilt cone are created by interactions with the surfaces of the bounding plates in thin cells. This effect occurs if $g_{1} \neq g_{2}$ and $\Delta(a, b) \leq 0$. When $\Delta(a, b)>0$, then expression $d \widetilde{G} / d a_{2}=0$ has one root in the $a_{2}$-space, and FLC is stable because free energy has one minimum (points $A$ and $D$ in Figure 2). If expression $d \widetilde{G} / d a_{2}=0$ has three roots, then bistabilities in FLC occur, and $\Delta(a, b)<0$ (see Figure 4 ).

Thus, bistable state of FLC is described by using the free energy potential and the cusp catastrophe model. The plot $\Delta(E)$ can help to determine the range of the electric field when bistability in FLC occurs.

\section{Conclusions}

The main result of this paper for theory of FLC is the justification of the relation between behavior parameters in the series expansion of azimuthal angle. For solution of this problem we considered equilibrium state of FLC in the "bookshelf" geometry when an electric field is applied perpendicular to the substrates. The proposed expansion for $\varphi$ helped us to reach the following numerical results: by using 
the torque balance equation and the bifurcation theory, one can readily establish the relationship between the behavior parameters $a_{i}$. The free energy potential given in (2) can be replaced by a functional with a priori known properties. The demo example explicitly shows bistability of FLC from the value of $E$ that corresponds to the break of zenithal anchoring.

\section{Conflict of Interests}

The authors declare that there is no conflict of interests regarding the publication of this paper.

\section{Acknowledgment}

This work was supported by the Russian Foundation for Basic Research (RFBR) under Project no. 14-02-97026.

\section{References}

[1] N. A. Clark and S. T. Lagerwall, "Submicrosecond bistable electro-optic switching in liquid crystals," Applied Physics Letters, vol. 36, no. 11, pp. 899-901, 1980.

[2] C. Wang and P. J. Bos, "Bistable C1 ferroelectric liquid crystal device for e-paper application," Displays, vol. 25, no. 5, pp. 187194, 2004.

[3] T. Poston and I. Stewart, Catastrophe Theory and Its Applications, Dover, New York, NY, USA, 2006.

[4] L. Gibelli and S. Turzi, "A catastrophe-theoretic approach to tricritical points with application to liquid crystals," SIAM Journal on Applied Mathematics, vol. 70, no. 1, pp. 63-76, 2009.

[5] G. Derfel, "Stationary states of the surface stabilized ferroelectric liquid crystal layers in electric field," Liquid Crystals, vol. 8, pp. 331-343, 1990.

[6] C. R. Ou, C.-M. Ou, C. H. Lin, and S. L. Young, "Catastrophic description of ferroelectric liquid crystal instabilities," Journal of Superconductivity and Novel Magnetism, vol. 23, pp. 917-921, 2010.

[7] I.-C. Khoo, Liquid Crystals, John Wiley \& Sons, Hoboken, NJ, USA, 2nd edition, 2007.

[8] M. Nakagawa, M. Ishikawa, and T. Akahane, "Dynamic responses of ferroelectric liquid crystals in the surfacestabilized geometry I," Japanese Journal of Applied Physics, vol. 27, no. 4, pp. 456-463, 1988.

[9] A. H. Nayfeh, Perturbation Methods, Wiley Classics Library, Wiley-Interscience, New York, NY, USA, 2000.

[10] P. Archer, I. Dierking, V. Görtz, and J. W. Goodby, "Probing the material properties and phase transitions of ferroelectric liquid crystals by determination of the Landau potential," The European Physical Journal E, vol. 25, pp. 385-393, 2008.

[11] A. Smith and I. W. Stewart, "Asymptotic solutions for an electrically induced Freedericksz transition in a wedge of smectic $\mathrm{C}$ liquid crystal," Journal of Physics A: Mathematical and General, vol. 39, pp. 11361-11382, 2006.

[12] L. Blinov, Structure and Properties of Liquid Crystals, Springer, 2011. 

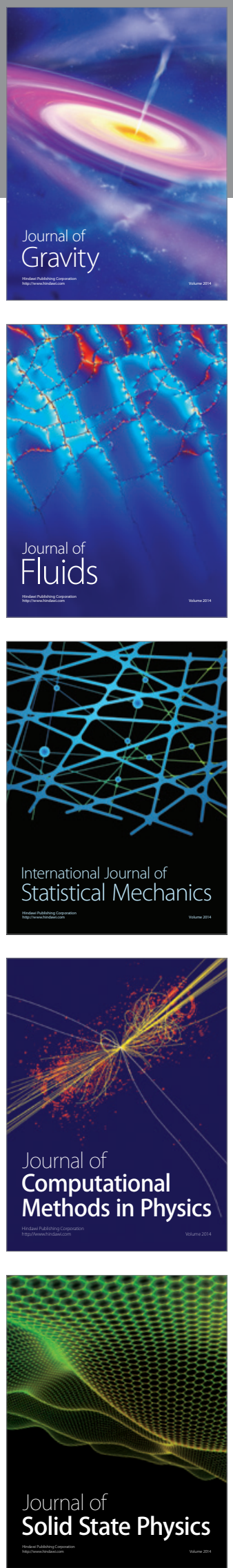

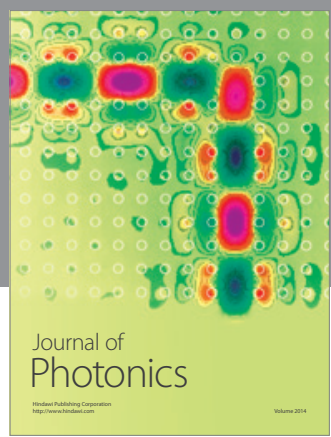

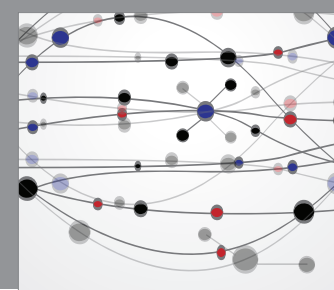

The Scientific World Journal

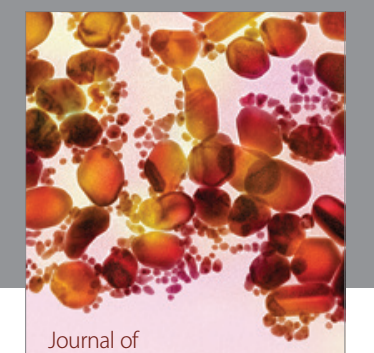

Soft Matter
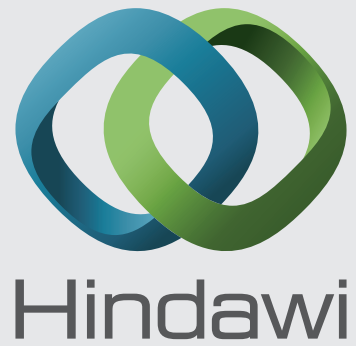

Submit your manuscripts at

http://www.hindawi.com
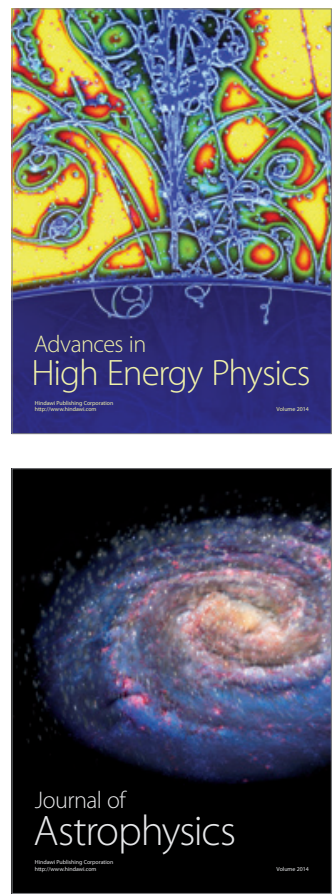
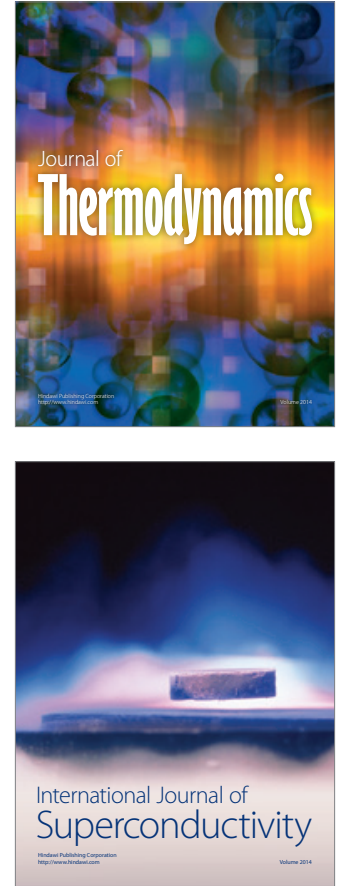
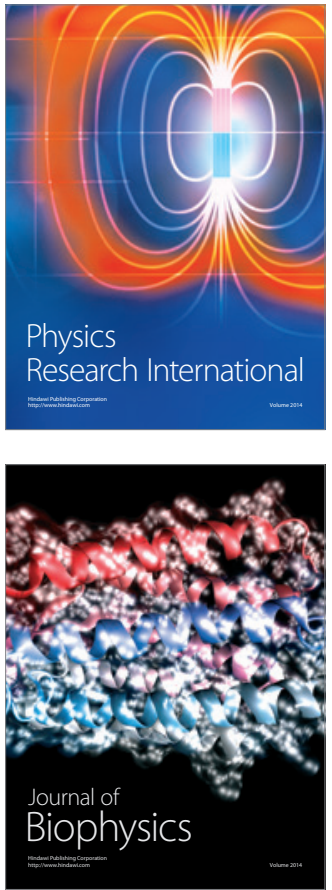
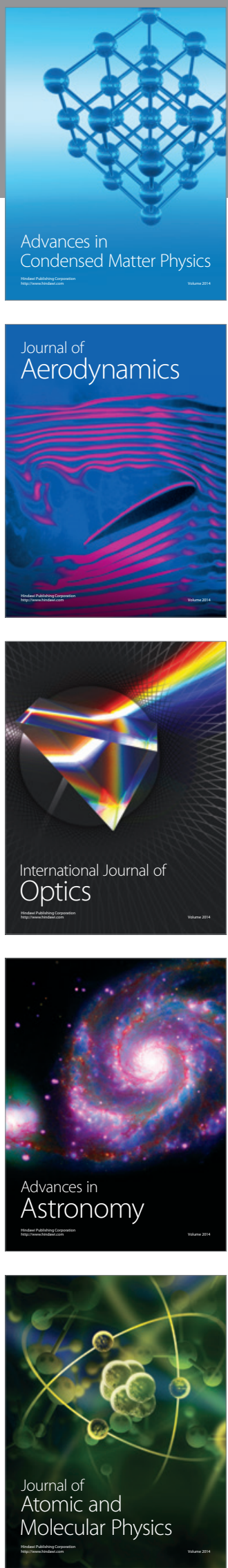